\title{
Distribution of coagulase-positive staphylococci in humans and dogs
}

\author{
Jurate Sleiniute, Jurate Siugzdaite \\ Lithuanian University of Health Sciences, Veterinary Academy, Department of Infectious Diseases, \\ Kaunas, Lithuania \\ Received February 3, 2015 \\ Accepted November 10, 2015
}

\begin{abstract}
Staphylococci form part of the normal flora of humans and a wide variety of animals. Some staphylococcal species also cause infections for human and household pets. The aim of this study was to investigate the prevalence of coagulase-positive staphylococci in humans and dogs within a common household. Coagulase-positive staphylococci could not be identified phenotypically in all cases. Molecular methods were used for the correct identification of pathogenic staphylococci. The prevalence of Staphylococcus aureus was identified in 26/72 (36.1\%) dog owners and in $4 / 61(6.6 \%)$ dogs. Staphylococcus pseudintermedius was isolated from three $(4.2 \%)$ humans, and from 28 dogs $(45.9 \%)$. Other coagulase-positive staphylococci species were not isolated in this study. Colonization of $S$. aureus in humans was significantly higher than in $\operatorname{dogs}(P<0.001)$. Staphylococcus pseudintermedius was more common in dogs compared to humans $(P<0.001)$. Staphylococcus pseudintermedius strains were isolated from dogs with clinical symptoms more frequently compared to healthy dogs $(P=0.02)$. Colonization of $S$. aureus in female dogs was significantly higher compared to male dogs $(P=0.045)$. The same staphylococci species were isolated from the dog and its owner in six households $(12 \%, 6 / 50)$.
\end{abstract}

Staphylococcus aureus, Staphylococcus pseudintermedius, bacteria, nasal, rectal

The importance of staphylococci as pathogens for humans and animals has been recognized for more than 100 years. There are about 40 species of staphylococci that differ in their ability to cause a risk to human and animal health, ranging from non-pathogenic to dangerous pathogens causing severe infections and being resistant to the treatment by most of the commonly applied antibiotics (Dobrindt et al. 2013). The coagulase test usually correlates well with staphylococci pathogenicity. The two major pathogenic staphylococci, Staphylococcus aureus and Staphylococcus pseudintermedius are coagulase-positive (Markey et al. 2013). Staphylococcus pseudintermedius has been described as a new coagulase-positive species of animals in 2005 (Devriese et al. 2005). Other coagulasepositive staphylococci are $S$. intermedius, S. schleiferi subsp. coagulans, S. hyicus, $S$. lutrae, and S. delphini (Sasaki et al. 2010).

Many healthy people may carry $S$. aureus as a part of their normal microflora in the nose, throat, perineum or skin (Narmeen et al. 2009). In the majority of cases, the bacteria do not cause disease. However, damage to the skin or other injury may allow the bacteria to overcome the natural protective mechanisms of the body, leading to infection. Skin infections including cellulitis, folliculitis, furuncles, impetigo and subcutaneous abscesses are the most common types of disease caused by S. aureus (Stulberg et al. 2002).

Staphylococcus pseudintermedius as normal microflora is usually isolated from dogs' skin and mucosa. This species are commensal organisms, but they are also a cause of disease such as pyoderma and otitis externa in dogs (Hanselman et al. 2009). Infection usually occurs when skin or mucosal barriers are affected by predisposing factors such as atopic dermatitis, medical and surgical procedures or immunosuppressive disorders (Bannoehr et al. 2009; 2012).

Address for correspondence:

Jurate Sleiniute

Department of Infectious Diseases Veterinary Academy

Lithuanian University of Health Sciences

Phone: +37067019744

Tilzes str. 18, LT-47181, Kaunas, Lithuania 
Staphylococcus pseudintermedius can cause clinical infections in humans as well, but these are rare and often misidentified as $S$. aureus (Wang et al. 2013).

In routine diagnostic bacteriology, $S$. aureus has long been differentiated from other species by means of a single test, most often coagulase or clumping-factor production (Devriese et al. 2005). However, S. pseudintermedius and other pathogenic staphylococci are also coagulase-positive. Extensive phenotypic testing or molecular identification methods are needed in order to identify these strains adequately (Devriese et al. 2005). Sasaki et al. (2007) devised a multiplex-PCR (M-PCR) method for species identification of coagulase-positive staphylococci by targeting the nuc gene locus.

Dogs and cats it seems to be the most popular pet animals in Europe, with nearly $24 \%$ of households having a dog (FEDIAF 2012). The relationship between companion animals and humans has changed throughout the years. While in the past dogs were maintained outside the households, today they are often kept inside the homes. Close physical contact by touching, petting and licking occurs at high frequency on the basis of the current perception of household pets as actual family members. Companion animals and humans can act as reservoirs of such bacteria (Lloyd 2007). Transmission of pathogenic staphylococci may occur between pets and owners. There have been several recent reports describing the carriage of indistinguishable coagulase-positive staphylococci strains in humans and animals (Van Hoovels et al. 2006; Hanselman et al. 2009).

The aim of this study was to expand the knowledge of pathogenic staphylococci occurring in humans and their household dogs.

\section{Materials and Methods}

The study involved 72 humans and $61 \mathrm{dogs}$ from 50 households. Animal owners completed a questionnaire on the data of each dog living in the same household. Information on the breed, age, sex and contact with the pet's characteristics were included in the questionnaire. The work was performed in compliance with Lithuanian animal welfare regulations (No. B1-866, 2012; No. XI-2271, 2012) and was approved by the Lithuanian Committee of the Veterinary Medicine and Zootechnics Sciences (Protocol No. 09/2012).

Dog owners were explained and given instructions on how to take samples correctly for self collection of nasal swabs. Nasal and rectal samples were collected from dogs of different ages, sexes and breeds, both healthy and with clinical symptoms (Table 1). The most common clinical signs were dermatitis, respiratory and gastrointestinal tract disorders. All swabs were placed in Amies medium TRANSWAB ${ }^{\circledR}$ (Medical wire, UK) and stored at $4{ }^{\circ} \mathrm{C}$ until processing.

Table 1. The characteristics of studied dogs.

\begin{tabular}{|c|c|c|c|c|c|c|c|c|c|}
\hline & \multirow{2}{*}{$\begin{array}{l}\text { Clinically } \\
\text { healthy }\end{array}$} & \multirow{2}{*}{$\begin{array}{l}\text { With clinical } \\
\text { signs of disease }\end{array}$} & \multicolumn{5}{|c|}{ Age } & \multicolumn{2}{|c|}{ Sex } \\
\hline & & & $\leq 1$ & $1-3$ & $4-5$ & $6-10$ & $\geq 10$ & female & male \\
\hline & & & years & & & & years & q & $\pi$ \\
\hline $\operatorname{Dogs}(n=61)$ & 51 & 10 & 7 & 20 & 9 & 20 & 5 & 31 & 30 \\
\hline
\end{tabular}

Swabs were placed in $2 \mathrm{ml}$ Tryptone Soya Broth (Oxoid, UK) for $24 \mathrm{~h}$ at $37{ }^{\circ} \mathrm{C}$. Enrichment culture was streaked on Mannitol Salt Phenol Red Agar (Sigma, USA) for staphylococci isolation and mannitol fermentation detection. Isolated staphylococci were stained by Gram's method. Catalase test with 3\% hydrogen peroxide was performed to determine if the gram positive cocci are Staphylococcus or Streptococcus species. Slide and tube coagulase tests with rabbit plasma (Coagulase Plasma EDTA, Biolife, Italy) were used to detect both bound and free coagulase. Clumping factor and protein A were detected by a rapid confirmatory latex agglutination test (Microgen Staph, Microgen Bioproducts, UK). All the isolates were tested for haemolysis on Tryptone soya agar (Oxoid, UK) supplemented with 5\% bovine blood. Biochemical tests were used for differentiating between Staphylococcus aureus and Staphylococcus pseudintermedius. A standardized plate method on the DNase agar (Sifin, Germany) was used for DNase activity detection. Fermentation of maltose was determined using violet agar plates (Liofilchem, Italy) with $1 \%$ of maltose. Baird Parker medium (BPA) (Liofilchem, Italy) was used for the detection of lecithinase and lipase activity of the coagulase positive staphylococci. All solid media were incubated for $24-48 \mathrm{~h}$ at $37^{\circ} \mathrm{C}$. 
Staphylococci isolates were genotypically confirmed by multiplex polymerase chain reaction (M-PCR). The DNA was extracted from overnight coagulase-positive staphylococci culture grown on Tryptone soya agar (Oxoid, UK) using 5\% solution of Chelex-100 (Sigma, USA). Several bacterial colonies were suspended in $500 \mu \mathrm{l}$ of Chelex-100 solution. After thermal lysis at $56^{\circ} \mathrm{C}$ for $30 \mathrm{~min}$ and $96{ }^{\circ} \mathrm{C}$ for $10 \mathrm{~min}$, suspensions were chilled on ice, centrifuged at $8,154 \mathrm{~g}$ for $2.5 \mathrm{~min}$ twice. Supernatant was used as DNA template.

Multiplex polymerase chain reaction (M-PCR) was carried out according to Sasaki's recommendations (Sasaki et al. 2009) with minor modifications.

The reaction mixture for PCR consisted of $3 \mu \mathrm{l}$ of DNA extract at a total volume of $25 \mu$ composed of $0.3 \mu \mathrm{l}(500 \mathrm{U})$ Taq DNA polymerase (MBI, Fermentas), $0.2 \mu$ l each primer (Table 2) (Grida Lab, Lithuania), $2 \mu 1$ (2 mM) dNTP mixture (MBI, Fermentas), $2.5 \mu \mathrm{l}(25 \mathrm{mM}) \mathrm{MgCL}_{2}$ (MBI, Fermentas), $2.5 \mu \mathrm{l}(1.25 \mathrm{ml}) 10 \times \mathrm{Taq}$ buffer with $\left(\mathrm{NH}_{4}\right)_{2} \mathrm{SO}_{4}$ (MBI, Fermentas), and $13.7 \mu$ l bidistilled water. Reaction mixtures were thermally cycled once at $95{ }^{\circ} \mathrm{C}$ for $2 \mathrm{~min} ; 30$ times at $95{ }^{\circ} \mathrm{C}$ for $30 \mathrm{~s}, 56{ }^{\circ} \mathrm{C}$ for $35 \mathrm{~s}$, and $72{ }^{\circ} \mathrm{C}$ for $1 \mathrm{~min}$; and then once at $72{ }^{\circ} \mathrm{C}$ for $2 \mathrm{~min}$ in thermocycler (G-STORM GS1, UK). DNA fragments were analyzed by electrophoresis in $1 \times$ Trisacetate-EDTA on a 1.2\% UltraPure agarose gel (Invitrogen, UK) stained with ethidium bromide. Images of gel were taken using the documentation system Molecular $\operatorname{Imager}^{\circledR}$ (Gel Doc ${ }^{\mathrm{TM}}$ XR, BioRad).

The prevalence of $S$. aureus and S. pseudintermedius in humans and dogs was calculated. The SPSS 13.0 statistical packet was used to analyze the data (Version 15, SPSS Inc., Chicago, IL). Comparisons were performed using chi-squared analysis, Fisher's exact and McNemars test using exact $P$-values. A $P$-value of $<0.05$ was considered significant for all comparisons.

Table 2. Oligonucleotide primers used for coagulase positive staphylococci identification.

\begin{tabular}{|c|c|c|c|}
\hline $\begin{array}{l}\text { Specific } \\
\text { elements }\end{array}$ & $\begin{array}{c}\text { Names of } \\
\text { oligonucleotide } \\
\text { primers }\end{array}$ & Sequence of oligonucleotide primers $5^{\circ} \rightarrow 3^{\text {c }}$ & $\begin{array}{l}\text { Lenght } \\
\text { of PCR } \\
\text { product }\end{array}$ \\
\hline S. aureus & $\begin{array}{l}\text { au-F3 } \\
\text { au-nucR }\end{array}$ & $\begin{array}{l}\text { TCGCTTGCTATGATTGTGG } \\
\text { GCCAATGTTCTACCATAGC }\end{array}$ & $359 \mathrm{bp}$ \\
\hline S. pseudintermedius & $\begin{array}{l}\text { psc-F2 } \\
\text { psc-R5 }\end{array}$ & $\begin{array}{l}\text { TRGGCAGTAGGATTCGTTAA } \\
\text { CTTTTGTGCTYCMTTTTGG }\end{array}$ & 926 bp \\
\hline S. intermedius & $\begin{array}{l}\text { in-F } \\
\text { in-R3 }\end{array}$ & $\begin{array}{l}\text { CATGTCATATTATTGCGAATGA } \\
\text { AGGACCATCACCATTGACATATTGAAACC }\end{array}$ & $430 \mathrm{bp}$ \\
\hline $\begin{array}{l}\text { S. schleiferi } \\
\text { subsp. coagulans }\end{array}$ & $\begin{array}{l}\text { sch-F } \\
\text { sch-R }\end{array}$ & $\begin{array}{l}\text { AATGGCTACAATGATAATCACTAA } \\
\text { CATATCTGTCTTTCGGCGCG }\end{array}$ & $526 \mathrm{bp}$ \\
\hline
\end{tabular}

\section{Results}

Staphylococci strains isolated from dogs and humans were characterized and identified using the most important biochemical tests. Some of the biochemical properties were weak or atypical for supposed staphylococci (Table 3). Staphylococcus spp. strains were isolated from 67 humans $(93.1 \%)$ and from $52 \mathrm{dogs}(85.2 \%)$. The coagulase-negative species were the most common of the isolates (Fig. 2). Significant higher $(P=0.035)$ number of coagulase-negative staphylococci were isolated from healthy dogs' samples $(47 \%)$ compared with sick dogs' nasal and rectal samples $(25 \%)$.

All $S$. aureus strains were positive by both tube and slide coagulase tests using sterile plasma as expected. However, $47 \% \mathrm{~S}$. pseudintermedius isolates produced bound coagulase. Both human and animal $S$. aureus and $S$. pseudintermedius isolates produced the presence of protein A by using agglutination test as shown in Table 3.

Our investigation showed that $31 \% \mathrm{~S}$. aureus strains isolated from human nasal cavity produced carotenoid pigment, this type of bacteria isolated from dogs were not producing yellow pigment. All coagulase-positive staphylococci isolated from animals and most of the $S$. aureus strains isolated from humans were characterized by DNase activity (Table 2). All $S$. pseudintermedius strains were positive for haemolysis, $17(65 \%)$ of the $S$. aureus 
Table 3. Biochemical characteristics of coagulase positive staphylococci isolated from humans and dogs.

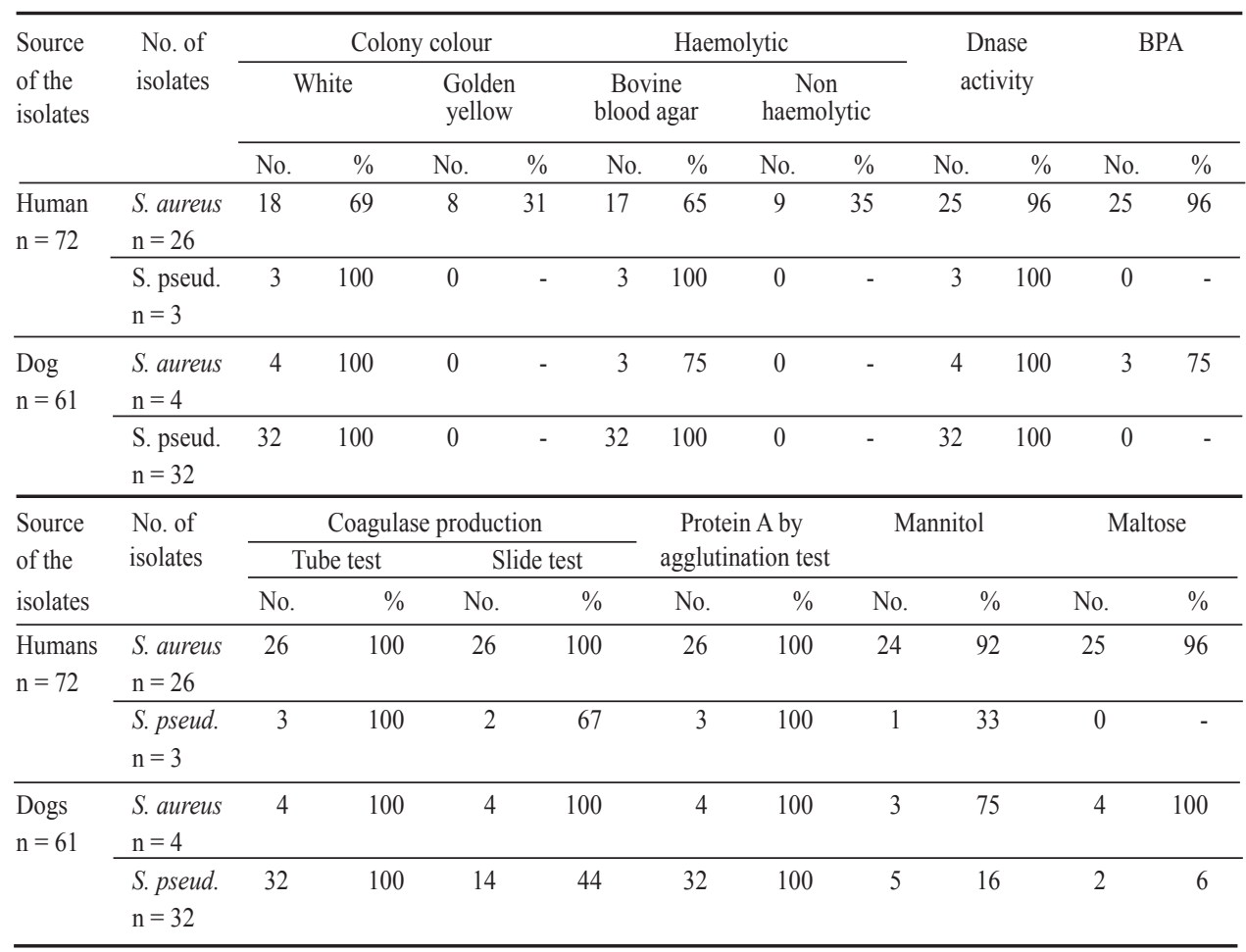

isolates from humans and from 3 dogs (75\%) showed beta-haemolysis on blood agar. We found that $25(96.2 \%)$ S. aureus strains isolated from humans and 3 (75\%) S. aureus isolates from the dogs showed lipase and lecithinase activity on BPA. Fermentation of sugars is shown in Table 3.

Two species of coagulase-positive staphylococci were identified by using M-PCR method. S. aureus and $S$. pseudintermedius showed a successful amplification of internal fragments with the expected sizes (359 bp and $926 \mathrm{bp}$ respectively) with the primer pairs specific for each species (Fig. 1).

The prevalence of $S$. aureus nasal colonization in healthy pet owners was identified in 26/72 (36.1\%) samples. Staphylococcus pseudintermedius was isolated from 3/72 (4.2\%) humans, these bacteria were isolated from their owned dogs as well.

Staphylococcus aureus strains were isolated from 6.6\% (4/61) dogs and more frequently from females than in males dogs $(P=0.045)$. Staphylococcus aureus commonly colonized canine nasal cavity $(75 \%, 3 / 4)$ compared with rectal colonization $(25 \%, 1 / 4)$ however, the difference was not significant $(P=0.6)$. All $S$. aureus strains were isolated from healthy $\operatorname{dogs}(P=0.37)$.

The prevalence of $S$. pseudintermedius in dogs was 45.9\% (28/61) - significantly higher than the prevalence of $S$. aureus $(P<0.001)$. Staphylococcus pseudintermedius strains were isolated from $90 \%(9 / 10)$ dogs with clinical symptoms and from $41.2 \%(21 / 51)$ healthy dogs, the difference was significant $(P=0.02)$. These bacteria were the most identified in the dogs with dermatitis $(55.6 \%)$. Staphylococcus pseudintermedius more commonly colonized canine rectum $(64 \%, 18 / 28)$ compared with nasal cavity colonization 


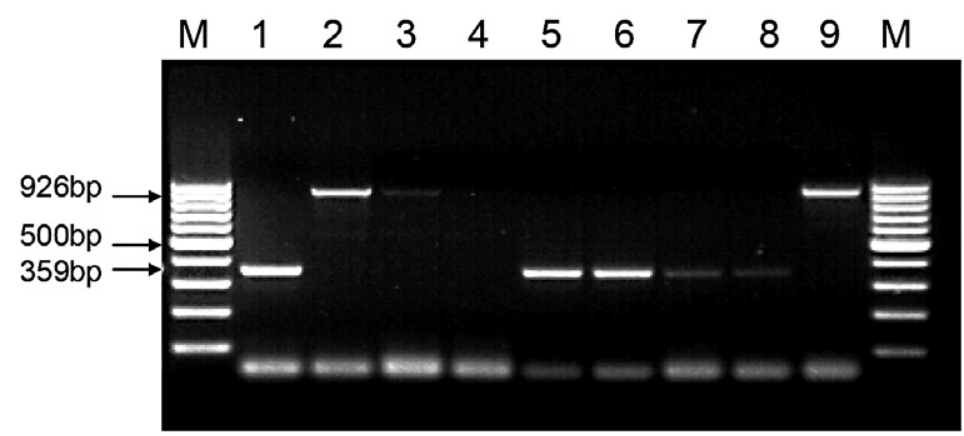

Fig. 1. Electrophoresis after M-PCR for species identification of coagulase-positive staphylococci on a $1.2 \%$ agarose gel. Lane $\mathrm{M}=$ GeneRuler TM 1000 bp DNA Ladder (MBI, Fermentas). Positive controls: lanes $1=S$. aureus (ATCC 9144); $2=S$. pseudintermedius (ATCC 49051). Lanes 5-8 $=$ S. aureus; 3; $9=$ S. pseudintermedius, $4=$ negative strain.

(43\%, 12/28), however, the value was not significant $(P=0.2)$. Dog's sex did not influence isolation of $S$. pseudintermedius $(P=0.9)$. Staphylococcus pseudintermedius was isolated from both sites - rectum and nose in two dogs (Fig. 2).

Colonization of $S$. aureus in humans was significantly higher than in dogs $(P<0.001)$. Staphylococcus pseudintermedius was more common in dogs compared to humans $(P<0.001)$.

Age, breed and keeping outside or inside did not influence the coagulase-positive staphylococcal colonization of the nasal cavity and rectum in dogs. Other coagulasepositive staphylococci species were not isolated in this study.

The same species staphylococci (three strains of $S$. aureus and three strains of $S$. pseudintermedius) were isolated from the dog and its owner in six households $(12 \%$, 6/50). All the humans, in whom the same staphylococcal species was found, responded in questionnaires that they do not wash their hands or do it sometimes after contact with pets, and $66.7 \%$ sleep together with their dogs in the same bed.

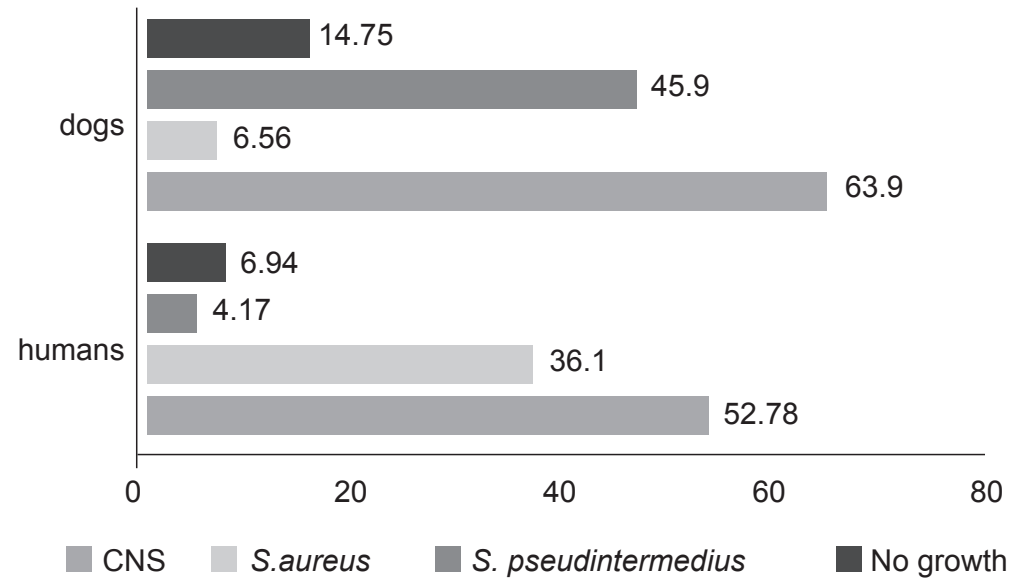

Fig. 2. Staphylococci isolated from humans and dogs. 


\section{Discussion}

Coagulase-positive staphylococci could not be identified phenotypically in all cases. Some of the biochemical properties were weak or atypical for certain staphylococci and it was difficult to determine the staphylococcus species correctly.

El-Jakee et al. (2008) assumed that the biochemical properties of $S$. aureus isolated from different sources did not differ significantly, and there is no special test providing the best differentiation. Thus, it can be concluded that we should always be aware of the existence of atypical $S$. aureus strains. These strains can easily be misclassified in routine bacteriology. Better diagnostic tools are needed for the identification of $S$. pseudintermedius, and to avoid misidentification with $S$. aureus and $S$. intermedius, according to researchers. Molecular methods must be used for the correct differentiation of coagulase positive staphylococci (Van Duijkeren et al. 2010; Savini et al. 2013).

Our study showed that staphylococci are widely spread among humans and dogs. The most common isolates were coagulase-negative staphylococci. The statistical analysis showed that these bacteria are more prevalent among healthy dogs. Identification of coagulase-positive staphylococci $S$. aureus and $S$. pseudintermedius was confirmed by using M-PCR method.

Staphylococcus aureus most commonly colonized the human nasal cavity, as expected. The frequency of $S$. aureus carriage in owners $36.1 \%$ was slightly higher than in other published reports. Staphylococcus aureus nasal colonization level 24\% found by Boost et al. (2008) was similar to the $27.7 \%$ carriage rate reported by Hanselman et al. (2009). Collaboration between researchers has identified a mechanism by which $S$. aureus colonizes the nasal cavity. The study shows that a protein located on the bacterial surface called clumping factor B has high affinity for the skin protein loricrin (Mulcahy et al. 2012).

Staphylococcus aureus is not regarded as a commensal organism of dogs. The prevalence of $S$. aureus colonization in dogs $(6.6 \%)$ was somewhat lower than identified by Hanselman et al. (2009) (14.3\%). Our results showed a higher prevalence of $S$. aureus in female dogs agreed with data previously published by Boost et al. (2008). Scientists believe that hormonal factors and behavioral differences between sexes may influence staphylococcal colonization.

Staphylococcus pseudintermedius is a veterinary pathogen that has seldom been described as an agent of human disease (Savini et al. 2013). Van Hoovels et al. presented the first clinical report of S. pseudintermedius infection in a human in 2006. Staphylococcus pseudintermedius colonization of the human nasal cavity has been rarely identified in our study (4.2\%). This species of bacteria was isolated from $4.1 \%$ of humans in a research performed in Canada (Hanselman et al. 2009). There are some case reports of methicillin-resistant Staphylococcus pseudintermedius (MRSP) infection in dog owners. The increasing number of dogs carrying MRSP constitutes a risk for pet owners to become colonized with MRSP, and MRSP infections in humans may also increase in the near future (Stegmann et al. 2010; Savini et al. 2013).

A high prevalence of $S$. pseudintermedius in dogs (45.9\%) was detected in our research. It was not surprising, Hanselman et al. (2009) reported that $S$. pseudintermedius was the most common in dogs as well; the results $(46.2 \%)$ were similar to our data. They found that rectal colonization $(60.7 \%)$ was significantly more common than nasal colonization $(23.0 \%)$. Our results showed that $S$. pseudintermedius were frequently isolated from the rectum $(64.0 \%)$. However, the value was not significant compared to nasal colonization $(43.0 \%)$. We found that $S$. pseudintermedius isolation depends on the dog's health $(P=0.02)$. Five dogs $(55.6 \%)$ in which this bacterium was isolated had dermatitis, other carriers had respiratory and digestive diseases. Fazakerley et al. (2009) found that 87.5\% 
dogs with atopic dermatitis were colonized with S.(pseud)intermedius compared to $37.2 \%$ of healthy dogs in a UK performed research.

Researchers from Ontario reported that concurrent human and dog colonization with $S$. aureus was demonstrated in $6.6 \%$ households, S. pseudintermedius were presented in $7.4 \%$ of households (Hanselman et al. 2009). We found similar results, S. aureus was isolated from the dog and owner in $6 \%$ households, and $S$. pseudintermedius in $6 \%$ households, as well. However, genotyping investigations are needed to evaluate these indistinguishable staphylococcal strains.

Research articles published before 2005 and a few years later described $S$. intermedius isolation from animals and humans. Previously, $S$. intermedius was considered to be responsible for most cases of the skin and ear infections in companion animals. However, using molecular phylogenetic analyses, research has demonstrated that isolates phenotypically identified as $S$. intermedius consist of three distinct species, including $S$. intermedius, $S$. pseudintermedius, and $S$. delphini. These species belong to the socalled Staphylococcus intermedius group (SIG). Importantly, it was discovered that $S$. pseudintermedius, not $S$. intermedius, is the common pathogen in dog (Bannoehr et al. 2009; Youn et al. 2011). It is likely that human and veterinary S. pseudintermedius isolates have been misidentified as $S$. aureus, $S$. intermedius, and $S$. delphini in the past (Sasaki et al. 2007; Savini et al. 2013).

Our study showed that staphylococci are widespread among humans and dogs. Two species of coagulase-positive staphylococci - Staphylococcus aureus and Staphylococcus pseudintermedius were identified by using multiplex PCR method. The carriage of Staphylococcus aureus in humans was significantly higher than in dogs. Staphylococcus pseudintermedius was more common in dogs. We found that this species of bacteria was often isolated from dogs with clinical symptoms. The same staphylococci species were isolated from the dog and its owner in several cases.

\section{References}

Bannoehr J, Franco A, Iurescia M, Battisti A, Fitzgerald JR 2009: Molecular diagnostic identification of Staphylococcus pseudintermedius. J Clin Microbiol 47: 469-471

Bannoehr J, Guardabassi L 2012: Staphylococcus pseudintermedius in the dog: taxonomy, diagnostics, ecology, epidemiology and pathogenicity. Vet Dermatol 23: 253-26

Boost MV, O’Donoghue MM, James A 2008: Prevalence of Staphylococcus aureus carriage among dogs and their owners. Epidemiology and Infection 136: 953-964

Devriese LA, Vancanneyt M, Baele M, Vaneechoutte M, De Graef E, Snauwaert C, Cleenwerck I, Dawyndt P, Swings J, Decostere A, Haesebrouck F 2005: Staphylococcus pseudintermedius sp. nov., a coagulase-positive species from animals. Int J Syst Evol Microbiol 55: 1569-1573

Dobrindt U, Hacker JH, Svanborg C 2013: Between pathogenicity and commensalism. In: Rosenstein R, Götz F: What distinguishes highly pathogenic staphylococci from medium- and non-pathogenic? Springer, Berlin, Heidelberg, 33-89 p.

Van Duijkeren E, Catry B, Greko Ch, Moreno MA, Pomba MC, Pyörälä S., Ruzauskas M, Sanders P, Threlfall EJ, Torren-Edo J, Törneke K 2011: Review on methicillin-resistant Staphylococcus pseudintermedius. J Antimicrob Chemother 66: 2705-2714

El-Jakee J, Nagwa AS, Bakry M, Zouelfakar SA, Elgabry E, Gad El-Said WA 2008: Characteristics of Staphylococcus aureus strains isolated from human and animal sources. Am Eurasian J Agric Environ Sci 4: 221-229

Fazakerley J, Nuttall T, Sales D, Schmidt V, Cartier SD, Hart CA, McEwan NA 2009: Staphylococcal colonization of mucosal and lesional skin sites in atopic and healthy dogs. Vet Derm 20: 178-184

Hanselman BA, Kruth SA, Rousseau J, Weese JS 2009: Coagulase positive staphylococcal colonization of humans and their household pets. Can J Vet 50: 954-995

Youn JH, Yoon JW, Koo HC, Lim SK, Park YH 2011: Prevalence and antimicrogram of Staphylococcus intermedius group isolates from veterinary staff, companion animals, and the environment in veterinary hospitals in Korea. J Vet Diagn Invest 23: 268-274

Lloyd HD 2007. Reservoirs of antimicrobial resistance in pet animals. Clin Infect Dis 45: 148-52

Markey B, Leonard F, Archambault M, Cullinane A, Maguire D 2013: Clinical veterinary microbiology. Ireland, Elsevier, 105 p. 
Mulcahy ME, Geoghegan JA, Monk IR, O`Keeffe KM, Walsh EJ, Foster TJ, McLoughkin RM 2012: Nasal colonisation by Staphylococcus aureus depends upon clumping factor B binding to the squamous epithelial cell envelope protein loricrin. PLOS Pathogens 8: e1003092

Narmeen SM and Jaladet, Jubrael MS 2009: Isolation and identification of S. aureus using classical and molecular methods. J Duhok Univ 12: 10-17

Sasaki T, Kikuchi K, Tanaka Y, Takahashi N, Kamata S, Hiramatsu K 2007: Reclassification of phenotypically identified Staphylococcus intermedius strains. J Clin Microbiol 45: 2770-2778

Sasaki T, Tsubakishita S, Tanaka Y, Sakusabe A, Ohtsuka M, Hirotaki S, Kawakami T, FukataT, Hiramatsu K 2010: Multiplex-PCR method for species identification of coagulase-positive staphylococci. J Clin Microbiol 48: $765-769$

Savini V, Barbarini D, Polakowska K, Gherardi G, Białecka A, Kasprowicz A, Polilli E, Marrollo R, Di Bonaventura G, Fazii P, D’Antonio D, Międzobrodzki J, Carretto E 2013: Methicillin-resistant Staphylococcus pseudintermedius infection in a bone marrow transplant recipient. J Clin Microbiol 51: 1636-1638

Stegmann R, Burbens A, Marinta CA, Perreten V 2010: Human infection associated with methicillin-resistant Staphylococcus pseudintermedius ST71. J Antimicrob Chemother 65: 2047-2048

Stulberg DL, Penrod MA, Blatny RA 2002: Common Bacterial Skin Infections. Am Fam Physician 66: 119-124

Van Hoovels L, Vankeerberghen A, Boel A, Van Vaerenbergh K, Beenhouwer H 2006: First case of Staphylococcus pseudintermedius infection in a human. J Clin Microbiol 44: 4609-4612

Wang N, Neilan AM, Klompas M 2013: Staphylococcus intermedius infections: case report and literature review. Infect dis rep 5: 6-11

FEDIAF (the European pet food industry federation) 2012: Facts \& figures. Available at: http://www.fediaf.org/ facts-figures/. Accessed May 3, 2014 